\title{
Molecular insight into the wetting behavior and amphiphilic character of cellulose nanocrystals
}

\author{
David C. Malaspina \\ ICMAB-CSIC,Campus UAB Bellaterra, Barcelona, Spain. \\ Jordi Faraudo \\ ICMAB-CSIC,Campus UAB Bellaterra, Barcelona, Spain.
}

\begin{abstract}
The study of nanocellulose is a field of growing interest due to its many applications and its use in the development of biocompatible and eco-friendly materials. In spite of the vast number of studies in the field, many questions about the role of the molecular structure in the properties of cellulose are still subject of debate. One of these fundamental questions is the possible amphiphilic nature of cellulose and the relative role of hydrogen bonding and hydrophobic effect on the interactions of cellulose. In this work we present an extensive molecular dynamics simulation study of this question by analyzing the wetting of cellulose with water and organic solvent, its interaction with hydrophilic and hydrophobic ions and its interaction with a protein (human epidermal growth factor, hEGF). We consider two characteristic cellulose crystal planes of I $\beta$ cellulose with very different roughness, different hydrogen bonding capability and different exposure of cellulose hydrophobic groups (the (010) plane which has exposed -OH groups and the (100) plane with buried -OH groups). Our results show that both surfaces are simultaneously hydrophilic and lipophilic, with both surfaces having very similar contact angles. In spite of the global similarity of wetting of both surfaces, the molecular details of wetting are very different and substantial local wetting heterogeneities (which strongly depend on the surface) appear for both solvents. We also observe a weak interaction of both surfaces with hydrophobic and hydrophilic solutes. These weak interactions are attributed to the simultaneous lipophilic and hydrophilic character of both (100) and (010) cellulose surfaces. Interestingly, we found a substantial interaction of both cellulose planes with polar and apolar residues of the hEGF protein.
\end{abstract}

\footnotetext{
* Corresponding author

Email address: jfaraudo@icmab.es (Jordi Faraudo)
} 


\section{Introduction}

Cellulose is one of the most abundant polymers on Earth and it has been used as a source for materials in the form of flax, hemp, and cotton fibers since prehistoric times 11. Nowadays, its nanoscale form, nanocellulose (i.e., cellulose with

5 at least one dimension in the nano-scale) and nanocellulose composite based materials are emerging as interesting candidates for the development of ecofriendly advanced materials to substitute petroleum-based materials [2, 3, 4]. This growing interest in the development of new cellulose and nanocellulose based materials is accompanied by a revamped interest in the understanding of the relation between the properties of the material, its chemical structure and its hierarchical supramolecular organization [5, 6, which are not well understood in such a complex material. Interestingly, fundamental physico-chemical questions such as the role of hydrogen bonding and hydrophobic interactions (which are common in many subfields of colloid and interface science) are believed to 15 play key roles in properties of cellulose and nanocellulose which are not fully understood [7] .

Chemically (see Figure 1), cellulose is a polysaccharide formed by linearly connected repeated units of anhydrocellobiose (i.e. a disaccharide of two glucose molecules linked together through an oxygen covalently bonded to $\mathrm{C} 1$ of one 20 glucose ring and $\mathrm{C} 4$ of the adjoining ring, the so-called $\beta$ (1-4) glycosidic bond). Cellulose materials are made by these polysaccharide chains organized in highly hierarchical structures [2, 5, 6].

Cellulose chains assemble in nano-fibrils (sheet-like elongated structures with typical diameters in the $\sim 10 \mathrm{~nm}$ scale) which also assemble in fibers that are the

25 common basic constituent of cellulosic materials. Fibrils are semi-crystalline, with regions of low order coexisting with crystalline domains. There are substantial variations in basic properties of the fibrils such as the degree of crystallinity, shape, length and diameter as well as crystal structure depending on its origin (bacterial cellulose or cellulose obtained from wood for example [6, 8]).

30 What are the basic molecular and supramolecular driving forces responsible for the hierarchical organization of cellulose? As shown in Figure 1, cellulose has an extensive network of inter-molecular and intra-molecular hydrogen bonds. The properties of cellulose have been traditionally explained in terms of these hydrogen bond chains. Cellulose has a hygroscopic character [9] and in 35 water tends to form hydrogel structures, a behavior which has been related to the presence of many polar groups in cellulose and its ability to form H-bonds. Intramolecular $\mathrm{H}$-bonds are believed to provide chain stiffness and intermolecular hydrogen bonds are usually invoked to justify the assembly of the linear polymers into fibrillar structures. However, a different perspective has been

40 proposed in the literature (the so-called Lindman hypothesis) [7, 10, 11, 12, which considers that the relevance of hydrogen bonding in cellulose has been overemphasized and points out the importance of hydrophobic interactions in cellulose (which may give a sort of amphiphilic character to cellulose). This proposed role of hydrophobic interactions, which is a controversial issue in the field

45 of cellulose [13, is based on molecular structure considerations and on a careful 


\section{a)}

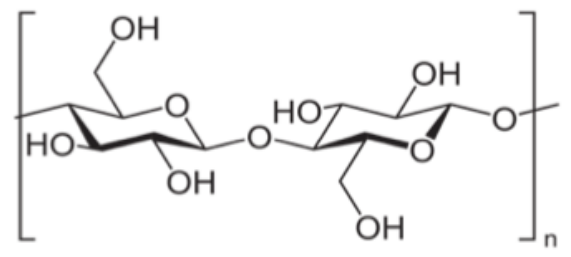

Cellulose monomer unit

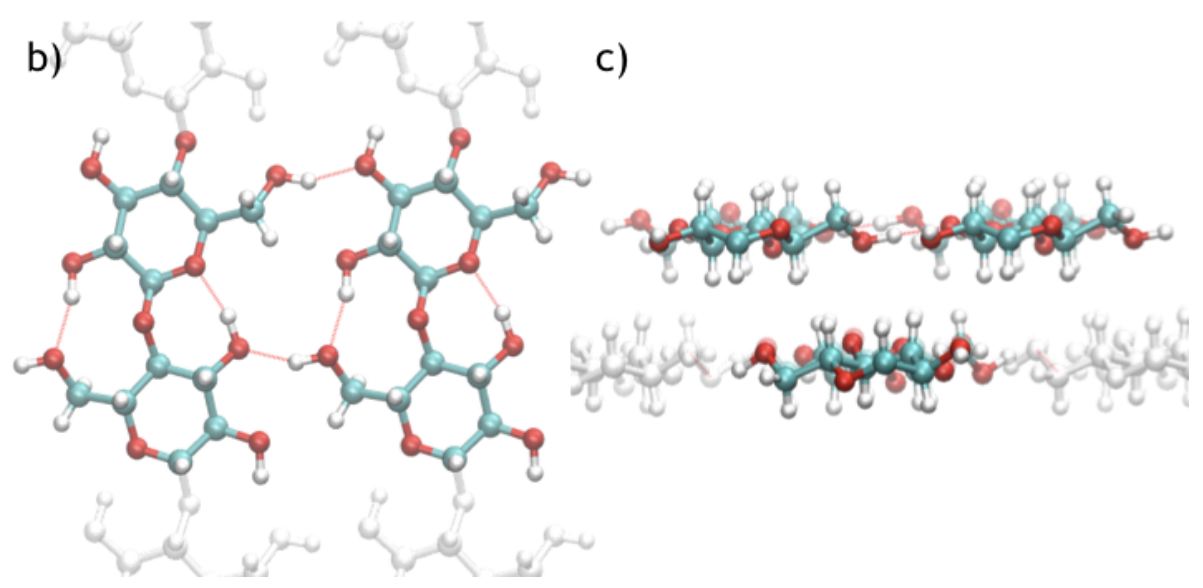

Figure 1: Molecular structure of cellulose. (a) Anhydrocellobiose repeated unit of cellulose, (b) Top view of two cellulose chains with indication of possible intra and inter molecular hydrogen bonds and (c) lateral view of the two cellulose chains shown in (b).

analysis of cellulose dissolution experiments [10. As seen in Figure 1] cellulose chains posses several hydrogen bond donors that give an hydrophilic character to the molecule, but at the same time pyranose rings in the molecule have a more hydrophobic character, so that cellulose molecules have an anisotropy in 50 their interactions, producing a complex competition between hydrophobic and hydrophilic character. Remarkably, it is very difficult to dissolve cellulose in both polar and apolar solvents. The solvents available for cellulose dissolution are of highly different nature which include unusual options (from simple or multicomponent mixtures, aqueous and organic media, inorganic and organic

${ }_{55}$ salts, with peculiar experimental conditions such as high and low temperatures or high and low $\mathrm{pH})$ 11, 12. This is complex pattern is a clear indication of the complexity of the interactions involving cellulose chains.

The fundamental question we want to address in this work is the connection between the molecular and supramolecular structure of cellulose and the role

60 of molecular-level interactions (hydrophobic effect, hydrogen bonding, ...) in cellulose-solvent and cellulose-molecule interactions. More specifically, we want to address theoretically, by using atomistic simulations, the question of the possible amphihilic, hydrophilic or hydrophobic character of cellulose starting from the chemical structure of cellulose and its crystal structure. 
The analysis of simulation results obtained from well-defined cellulose crystal structures in situations as diverse as wetting from different solvents or the interaction with different molecules (with different solvation characteristics and different hydrogen bonding capability) will allow us to discuss in full detail the roles of hydrophobic and H-bonding capabilities of cellulose at different scales.

70 The paper is organized as follows. In section 2, we will briefly summarize basic aspects of atomistic modelling of cellulose. In section 3 , we will consider MD simulations of wetting of cellulose crystals by different types of solvents (more specifically, we consider a droplet of water and a droplet of an organic solvent onto a cellulose crystal at ambient temperature). In section 4 , we consider the

75 interaction of cellulose with suspensions in water containing solutes of different hydrophobic or hydrophilic character and of different size (from small molecules to a protein). We end-up the paper with conclusions and perspectives (Section $5)$.

a)

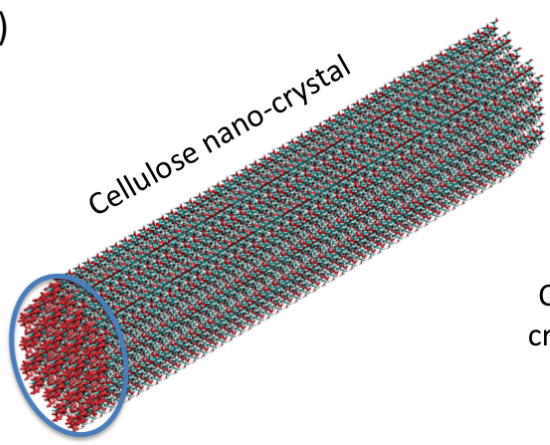

c)

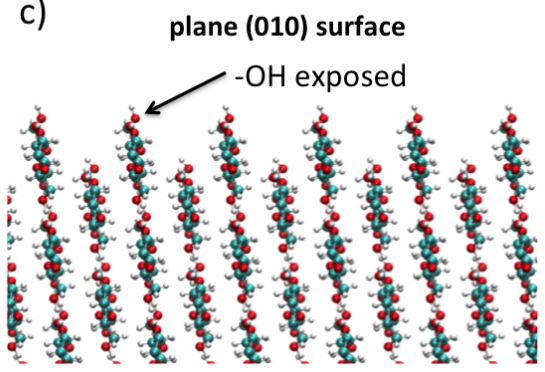

b)
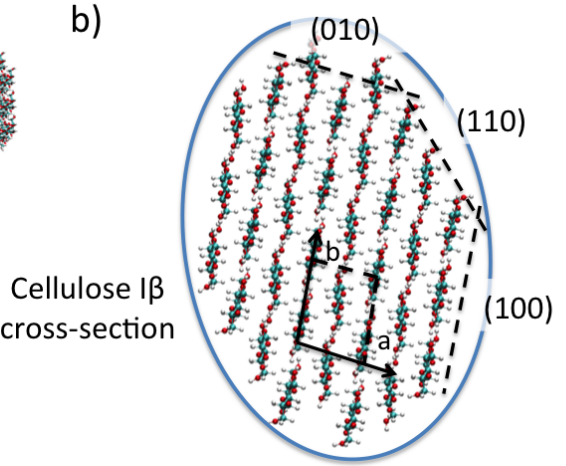

d)

plane (100) surface

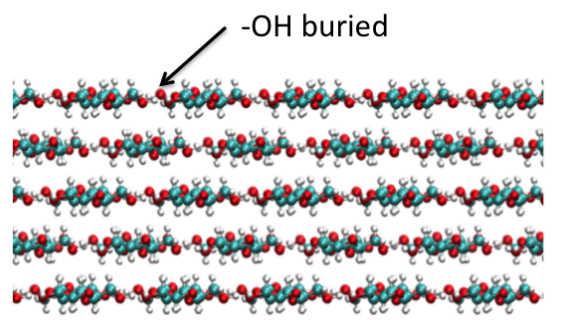

Figure 2: Atomistic modeling of $\mathrm{I} \beta$ cellulose (molecular representation and coloring uses the CPK convention). a) Nanocrystal (from proposed maize fibril shape 14]) with $\approx 4 \mathrm{~nm}$ diameter and $\approx 12 \mathrm{~nm}$ length (18144 atoms) generated with the "cellulose-builder" toolkit [15]. b) Cross section of (a) indicating the different crystallographic planes. c) Detail of the (010) plane in (b) with indication of surface - $\mathrm{OH}$ groups that are exposed and available for interaction for other molecules and solvents. d) Detail of the (100) plane in (b) indicating the location of the surface - $\mathrm{OH}$ groups involved in intramolecular hydrogen bonding. Image made with VMD [16. 


\section{Atomistic modeling of Cellulose} ing atomistic computer simulations of materials and their interactions with molecules and solvents under given thermodynamic conditions (temperature, pressure). Conceptually, the method is simple. It is based on the fact that at ordinary conditions (not under extreme temperatures or pressures), the motion cally the Newton laws of motion in a computer. This allows the prediction of supramolecular properties starting from an atomistic description of the system of interest.

The method requires the formulation of an atomistic model of the system

90 with an atomistically detailed structure and a method for the calculation of all interactions between atoms of the system, which in principle can be done at different levels of theory, ranging from quantum mechanical electronic structure methods to analytical force fields (which can be of semi-empirical origin or fitted from electronic structure calculations [18).

95

Atomistic simulations of a full fibril containing both crystalline and amorphous regions are not possible, since in order to be realistic they will require the consideration of system sizes of the order of microns [6] which are beyond the possibilities of atomistic simulations. Therefore, atomistic MD simulations focused on fully crystalline fragments of fibrils (elementary nanofibrils or nanocrysdimensions of the crystals in the simulations are in the order of $\approx 10 \mathrm{~nm}[19,20$. Simulations of larger structures are possible by considering MD simulations of coarse-grained (not atomistic) models [21, 22.

The first ingredient needed in MD simulations is the generation of atomic coordinates of the initial structures to be employed in the simulations. In the case of cellulose, this can be done using for example the "cellulose-builder" computational toolkit developed in Ref [15] which generates atomic coordinates of nanofibrils of any length with any of the known crystal structures of cellulose ( $\mathrm{I} \alpha, \mathrm{I} \beta, \mathrm{II}$, and $\mathrm{III}_{I}$ polymorphs 23 ). This tool can be combined with other packages for decoration of the cellulose fibrils with hemicellulose and lignin for simulations in the case of plant cellulose. An example of a nanofibril with the $\mathrm{I} \beta$ crystal structure generated using this tool is shown in Figure $2 a$.

In the interactions of cellulose with solvents and molecules, the properties of the cellulose surface play an essential role. As shown in Figure2 $2 \mathrm{~b}$, different sur-

115 faces planes with different $\mathrm{H}$-bond capability can be obtained from crystalline cellulose I $\beta$ 24. Plane (010) I $\beta$ display the highest accessibility for hydrophilic groups (-OH) and highest surface roughness (figure 2k) while plane (100) I $\beta$ display the lowest accessibility for hydrophilic groups and lowest surface roughness (figure2d). Plane (110) has an intermediate character between planes (010) and

groups accessibility and surface roughness 24 . Therefore, simulation studies of cellulose interactions with molecules and solvents should take into account this structural diversity in the character of cellulose surfaces. 
Concerning the modelization of the atomic interactions, there are several For example, in Ref 25] MD simulations using Gromos force field were employed to study the thermal expansion and the Young modulus of $I \beta$ cellulose obtaining a good agreement with experimental data. However, later simulations with longer simulation times employing also the Gromos force field indicated limita130 tions in reproducing the known crystal structures of cellulose [26]. In the same study, better results were obtained by using CHARMM C35 or GLYCAM06 force fields. A more recent, exhaustive comparison 19 between different wellknown molecular force fields (CHARMM C35, GLYCAM06 and CSFF) shows that these three force fields correctly predict the (experimentally known) crystal the modelization of atomic interactions, it is possible to perform atomistic MD simulations of cellulose crystals and their interactions with different solvents and with other molecules at the desired temperatures and pressures.

In fact, this MD force field methodology described above has been employed water and the effect of different factors (chain length, ionization state) has been studied in Ref [27] using CSFF forcefield for cellulose and TIP3P model for water and in Ref [28] using the Glycam forcefield for cellulose and the TIP4P/2005 model for water. The interaction of $\mathrm{I} \beta$ crystalline cellulose with water has field for cellulose and TIP3P water model was employed in Refs [29, 30, the PCFF force field for cellulose and SPC model for water was employed in Refs [31, 24 and Glycam06 forcefield for cellulose and TIP3P model for water was employed in Ref 32. The interaction of cellulose crystals with peptides has also been considered in Ref 30, modeling the cellulose and the protein with the CHARMM forcefield and the water with the TIP3P model. Concerning simulations in other solvents, in Ref [33] the authors performed MD simulations of a cellulose nanocrystal in a water/octane emulsion using CHARMM force field for cellulose and octane and the TIP3P model for water. The transfer of a 155 cellulose chain from the surface of a cellulose crystal surface towards bulk liquid (water or organic solvent) was simulated in Ref [34, using the GROMOS96 45A4 force field for cellulose, a simple Lennard-Jones liquid model for the organic solvent and the SPC model for water. There are also MD simulation studies of the interaction of cellulose with molecules and materials in water solvent. For example, Chen et al. 35 considered the interaction of urea in water with cellulose and Alqus et al. [36] considered the interaction of cellulose covered with graphene in water.

Without entering into the details of the results of all these diverse computational studies, we can say that they confirm the view that in cellulose crystals 165 both hydrogen bonding capability and hydrophobic interactions play a substantial role. Heterogeneity is also important, in the sense that these studies show a significant local dependence of hydrogen bonding and hydrophobic interactions.

We will further test this view here, by considering MD simulations of a I $\beta$ cellulose nanocrystal (Figure 2k, d) in several different situations, employing the 
Table 1: Summary of wetting simulations (solvent droplet over a surface). We indicate the name for each system, cellulose crystal plane, number of solvent molecules, total number of atoms, simulation time, and simulation box size.

\begin{tabular}{llccccc}
\hline Name & Solvent & $\begin{array}{c}\text { Cellulose I } \beta \\
\text { crystal plane }\end{array}$ & $\begin{array}{c}\text { Solvent } \\
\text { molec. }\end{array}$ & $\begin{array}{c}\text { Total } \\
\text { atoms }\end{array}$ & $\begin{array}{c}\text { Sim. } \\
\text { time }\end{array}$ & $\begin{array}{c}\text { Sim. box } \\
(\mathrm{nm} \times \mathrm{nm} \times \mathrm{nm})\end{array}$ \\
\hline S1 & water & $(010)$ & 884 & 28,736 & $20 \mathrm{~ns}$ & $9.4 \times 12 \times 10.5$ \\
S2 & water & $(100)$ & 884 & 33,776 & $20 \mathrm{~ns}$ & $12 \times 9.8 \times 10.3$ \\
S3 & tetradecane & $(010)$ & 83 & 28,852 & $20 \mathrm{~ns}$ & $9.3 \times 16 \times 10.5$ \\
S4 & tetradecane & $(100)$ & 83 & 33,892 & $20 \mathrm{~ns}$ & $14 \times 9.8 \times 10.3$ \\
\hline
\end{tabular}

same model for the cellulose crystal to study the interaction of its (010) and (100) facets with different solvents (water and organic solvent) and different molecules (with different solvation characteristics and different hydrogen bonding capability). The analysis of MD results obtained from well-defined cellulose crystal structures in these diverse situations will allow us to discuss in full de-

175 tail the roles of hydrophobic and H-bonding capabilities of cellulose at different scales.

In the simulations reported in the following sections, we always employ the CHARMM36 force field for modeling cellulose [37] since this general force field allows also the simulation of different solvents 38 and also proteins and ions in solution [39, making possible the study of the interaction of the interaction of cellulose crystals with them. For water, we will employ the TIP4P/2005 model [40] which is the water model that (up to now) best capture hydrogen-bonding features of liquid water at all temperatures and pressures [41] and also has the best performance in reproducing water properties (see 42] for a detailed com-

185 parison between the performance of different water models). Further technical details are given in the Appendix.

\section{Wetting of Cellulose: water and tetradecane}

In this section, we consider MD simulations of wetting of (010) and (100) surfaces of I $\beta$ cellulose by water and by an apolar solvent (tetradecane). We consider these two surfaces since, as discussed in the previous section, they have very different hydrogen bonding capabilities, being the (010) plane the one with the highest accessibility for hydrophilic groups (-OH) and highest surface roughness (see figure 22) while the plane (100) has the lowest accessibility for hydrophilic groups and lowest surface roughness (see figure $2 \mathrm{~d}$ ).

The details of these four simulations (two surfaces and two solvents) are summarized in Table 1. In the simulations, we initially place a solvent (water or tetradecane) nanodroplet of about $3 \mathrm{~nm}$ size near the surface ((010) or (100)). The results were analyzed once the droplet reaches an equilibrium configuration over the surface. The results for both surfaces are shown in Figure 3 for water and in Figure 4 for tetradecane.

The most important result of our simulations is that both surfaces show wetting behavior that corresponds simultaneously to hydrophilic and lipophilic 

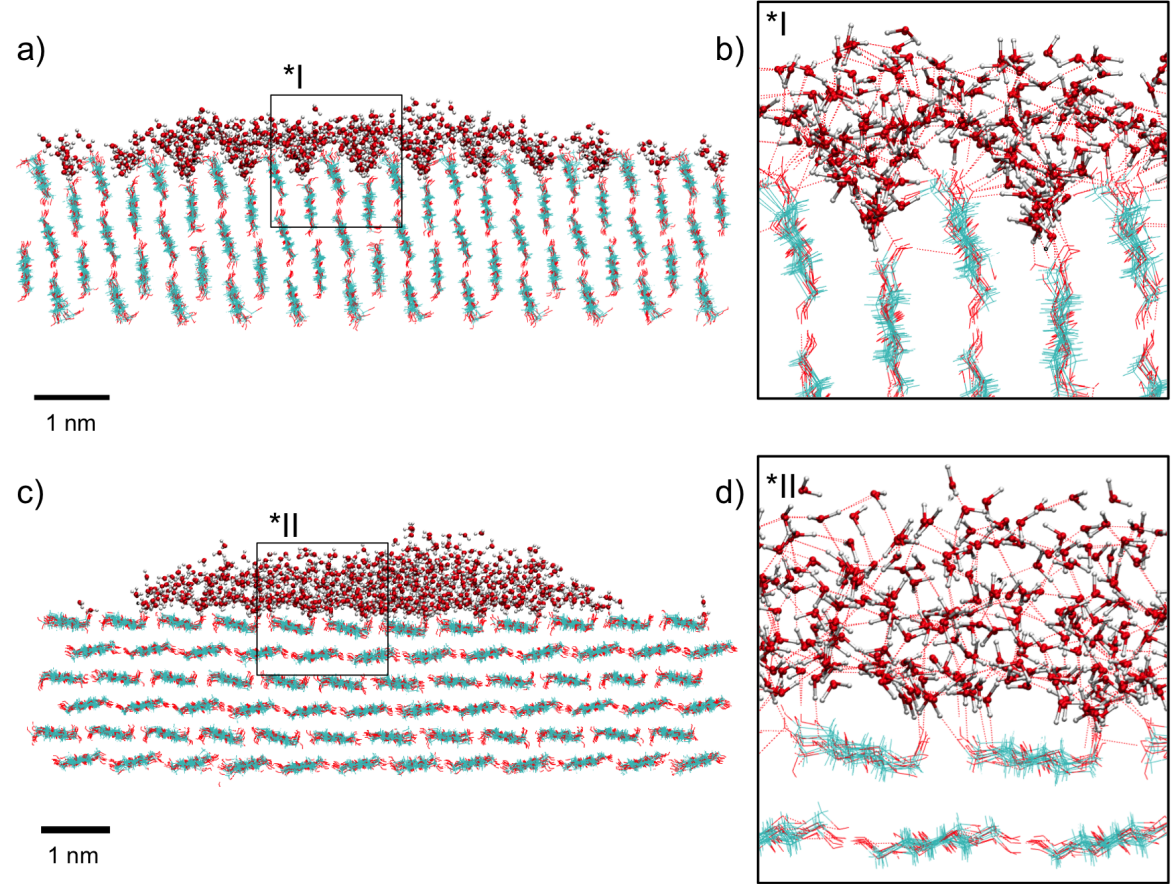

e)

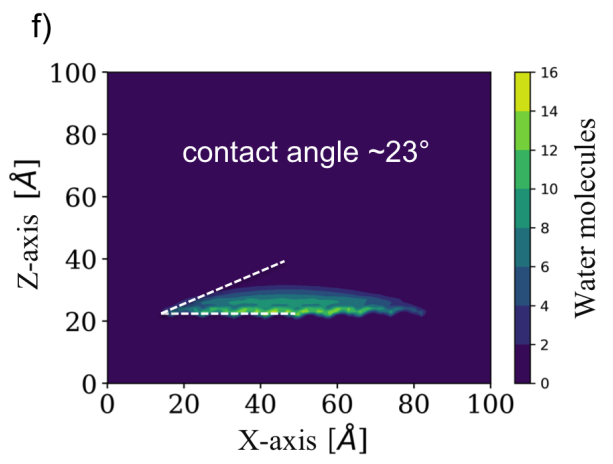

Figure 3: Cellulose wetting behavior with water. a) Cellulose plane (010) wet by a nanodroplet of water, observed from the side view. b) Detail of the hydrogen bonds formed. c) Cellulose plane (100) wet by a nanodroplet of water, observed from the side view. d) Detail of the hydrogen bonds formed. e) 2-D density profile of water molecules on plane (010), side view. Resulting contact angles are indicated in the figure. d) 2-D density profile of water molecules on plane (100), side view. Resulting contact angles are indicated in the figure.

behavior.

In the case of wetting by water simulations ( $\mathrm{S} 1$ and $\mathrm{S} 2$ in table1), we observe 205 the formation of a well-defined water droplet for both surfaces (see snapshots in Figure 3a and Figure c). The contact angles in the simulations are calculated from the average 2 -D water density profiles of the droplet. We obtain a contact 
angle of $\approx 16^{\circ}$ for the $(010)$ plane and $\approx 23^{\circ}$ for the $(100)$ plane, as seen in Figures 3 , and 3 .

It is interesting to compare our results with previous simulation and experimental results, which showed mixed conclusions. Early simulations 31 with the older SPC water model and the PCFF force field for cellulose predicted a high contact angle $\left(95^{\circ}\right)$ for plane (100) and a small contact angle $\left(46^{\circ}\right)$ for plane (110) (this plane has an intermediate character between plane (100) and 215 plane (010), see Figure 2). More recent MD results 30 using also CHARMM forcefield but with the TIP3P water model (instead of the TIP4P/2005 model employed here) predicted a contact angle of $25^{\circ}$ for water with cellulose plane (100), very similar to our results shown in Figure 3 ; (no results were reported for other planes in this case).

Experimental results show a wide variability in reported contact angles 43. 44, 45, due to many practical issues (specific experimental technique or protocol employed, type of sample, ...). Advancing and receding contact angles for water/air of $19^{\circ}$ and $12^{\circ}$ were observed for bacterial cellulose in Ref. 43. In Ref [4], time-dependent contact angles were observed for crystalline cellulose 225 which started at $45^{\circ}$ and were observed to decrease up to $32^{\circ}$. Time dependent contact angles were also observed for cellulose fibers [45, starting at $25^{\circ}$ and decreasing up to $10^{\circ}$. Besides the variability associated with these results, we can say that experimental contact angles are consistent with the hydrophilic character obtained in our simulations.

${ }_{230}$ In the case of wetting by tetradecane (S3 and S4 in table 1), we observe that the organic solvent spreads completely over both surfaces so the observed contact angles are zero (see Figure 4). Up to the best of our knowledge, there are no previous wetting simulations of cellulose by organic solvents. Also, we have not found experiments on wetting of crystalline nanocellulose by organic solvents. There are of course reports on wetting of wood by different liquids, which may be related to the wetting properties of cellulose. Interestingly several organic liquids completely wet wood with contact angles of zero 46.

There are also recent studies that highlight the amphiphilic behavior of cellulose. For example, Kalashnikova et al [47] studied the stabilization of oil/water 240 emulsion by cellulose crystals. They found that crystals with low surface charge groups favor the stability of emulsions suggesting that the amphiphilic nature of cellulose is the main driving force for the stabilization. MD simulations 33 of a cellulose crystal in oil/water emulsion also support this interpretation.

Our simulations presented here suggest that the proposed amphiphilic behavior (which suggests the presence of hydrophilic and hydrophobic regions) can be better described as a simultaneous hydrophilic and lipophilic behavior. All surfaces of cellulose crystal present this dual hydrophilic and lipophilic behavior. Noting that the contact angles for the organic solvent are smaller than the contact angles for water, we can also say that cellulose has larger affinity for 250 organic solvents than for water (is more lipophilic than hydrophilic).

Although the wetting behavior is the same for both (010) and (100) surfaces and both solvents (in the sense that both behave as hydrophilic and lipophilic), there are substantial differences in the molecular organization of the solvents at 

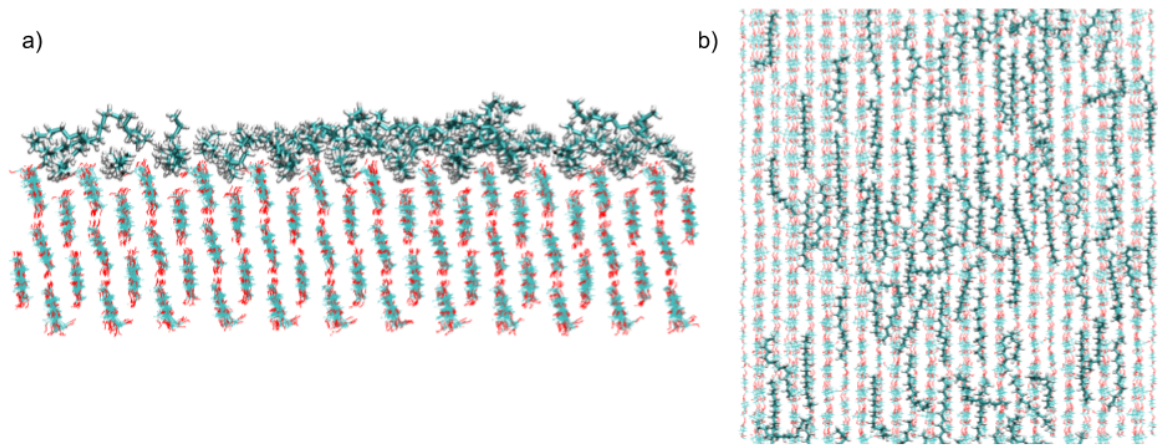

c)
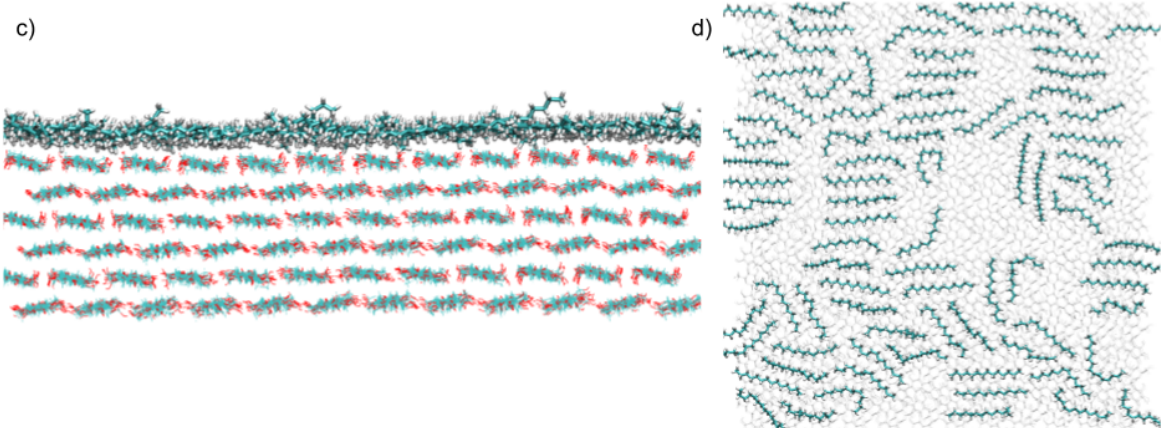

Figure 4: Cellulose wetting behavior with organic solvent. Cellulose plane (010) wet by a nanodroplet of tetradecane, observed from: a) side view and b) top view. Cellulose plane (100) wet by a nanodroplet of tetradecane, observed from: c) side view and d) top view.

the interface with each of these surfaces. In the case of water at the (010) surface (Figure $3 \mathrm{a}, \mathrm{b}$ ), we observe the penetration of water in the interstices between cellulose molecules, due to the high roughness of the surface. We also observe the formation of hydrogen bonds between water molecules and the exposed surface -OH groups of cellulose. In the case of the (100) plane (Figure 3 c, d), we observe the formation of water structures at the interface, with characteristic empty gaps on top of the apolar rings of cellulose. This peculiar ordering of water at plane (100) I $\beta$ has been also reported in several previous MD simulations 29, 32.

We also observe the formation of hydrogen bonds between water and the -OH groups of the interface, groups which initially were not accessible to water. Comparing the initial crystal structure shown in Figure $2 \mathrm{~d}$ with the structure obtained in presence of a water droplet (Figure $3 \mathrm{~b}$ ), we see that cellulose molecule-molecule $\mathrm{H}$-bonds have been replaced by cellulose-water H-bonds.

We recall that, in spite of these molecular-level differences in the organization of water molecules, the contact angles in both cases are very similar (see Figure 35). It is also clear that the wetting behavior of water at both surfaces 270 has heterogeneities. This may reflect an heterogeneous character of the interactions at the interface which may impact the interaction with ions with different degrees of hydration or molecules with hydrophobic and hydrophilic patches as 
Table 2: Summary of simulations with a molecule in water interacting with a cellulose surface. In all cases the molecules are negatively charged and the system is globally neutral, being the counterions $\mathrm{Na}^{+}$.

\begin{tabular}{llccccc} 
Name & Molecule & $\begin{array}{c}\text { Cellulose } \mathrm{I} \beta \\
\text { crystal plane }\end{array}$ & $\begin{array}{c}\text { Water } \\
\text { molec. }\end{array}$ & $\begin{array}{c}\text { Total } \\
\text { atoms }\end{array}$ & $\begin{array}{c}\text { Sim. } \\
\text { time }\end{array}$ & $\begin{array}{c}\text { Sim. box } \\
(\mathrm{nm} \times \mathrm{nm} \times \mathrm{nm})\end{array}$ \\
\hline $\mathrm{S} 5$ & $\mathrm{CO}_{3}^{2-}$ & $(010)$ & 2,260 & 15,094 & $190 \mathrm{~ns}$ & $4.6 \times 4.1 \times 5.9$ \\
$\mathrm{~S} 6$ & $\mathrm{CO}_{3}^{2-}$ & $(100)$ & 2,289 & 15,210 & $190 \mathrm{~ns}$ & $4.9 \times 4.1 \times 5.7$ \\
$\mathrm{~S} 7$ & $\mathrm{Ph}_{4} \mathrm{~B}^{-}$ & $(010)$ & 2,431 & 15,818 & $170 \mathrm{~ns}$ & $4.6 \times 4.1 \times 6.2$ \\
S8 & $\mathrm{Ph}_{4} \mathrm{~B}^{-}$ & $(100)$ & 2,859 & 17,530 & $170 \mathrm{~ns}$ & $4.9 \times 4.1 \times 6.5$ \\
S9a & $\mathrm{EGF}$ & $(010)$ & 24,665 & 124,665 & $83 \mathrm{~ns}$ & $9.5 \times 9.7 \times 10.3$ \\
S9b & $\mathrm{EGF}$ & $(010)$ & 24,198 & 122,797 & $120 \mathrm{~ns}$ & $9.5 \times 10.4 \times 9.2$ \\
$\mathrm{~S} 10 \mathrm{a}$ & $\mathrm{EGF}$ & $(100)$ & 25,318 & 132,317 & $78 \mathrm{~ns}$ & $10 \times 9.8 \times 10.3$ \\
$\mathrm{~S} 10 \mathrm{~b}$ & $\mathrm{EGF}$ & $(100)$ & 24,761 & 130,089 & $140 \mathrm{~ns}$ & $9.9 \times 10.4 \times 9.2$ \\
\hline
\end{tabular}

proteins. This aspect will be investigated in the following section.

The arrangement of tetradecane in both surfaces is also different, as seen in Figure 4. In the case of the (010) surface, tetradecane molecules follow the stripes defined by the surface roughness, while in the case of the (100) surface, tetradecane molecules tend to assembly in clusters with local order.

\section{Interaction of Cellulose with molecules in water}

The results obtained in the previous section emphasize the presence of heterogeneities in the wetting of cellulose by water (see Figure 3). A way to probe the effect of these molecular-scale heterogeneities is to investigate the interaction of cellulose with molecules. We will consider the case of small molecules with sizes similar to those observed in the heterogeneities and the case of a protein, which have patches with hydrophobic and hydrophilic regions. These simulations are summarized in Table 2

We will start by considering the interaction of molecular ions with cellulose. It is known that the interaction of ions with surfaces and interfaces is dominated by its hydration shell (which determines its hydrophobic or hydrophilic character) and the hydrophobic or hydrophilic character of the surface [48, 49, 50, giving the so-called Hoffmeister series (which classify ions with the same charge and different hydration).

We will consider here two extreme cases, carbonate ions $\left(\mathrm{CO}_{3}^{2-}\right)$ and tetraphenyl borate $\left(\mathrm{Ph}_{4} \mathrm{~B}^{-}\right)$ions. Carbonate ions are strongly hydrophilic [48]. They have an interaction with water molecules stronger than the water-water interaction 295 and they tend to retain their hydration shell [51. Due to this strong interaction they tend to be repelled from the air/water interface [48] and attracted to hydrophilic surfaces. On the other hand, $\mathrm{Ph}_{4} \mathrm{~B}^{-}$is a highly hydrophobic organic ion. It has a strong tendency to accumulate to lipophilic/hydrophobic surfaces, onto hydrophobic regions of polymers and at hydrophobic patches of proteins, as shown in several experimental and simulation studies [49, 50, 52, 53]. In 
both cases, we include in the simulations $\mathrm{Na}^{+}$as a counterion which is a weakly hydrophilic ion 50.

Hence, our interest now is to determine how is the interaction of these ions with I $\beta$ cellulose, which according to our wetting simulations reported in the previous section, is both lipophilic and hydrophilic.

The results are shown in Figure 5 for carbonate and in Figure 6 for tetraphenyl borate. Both ions show a weak interaction with the (010) and the (100) plane of I $\beta$ cellulose.

Let us discuss first the results for the case of the carbonate ion and the (010) surface. As shown in Figure 5a, there is a depletion of this ion from the interface. The configuration of maximum approach of the ion at the interface is shown in Figure 5b. In this case, the maximum approach to the surface is made in a way that the two $\mathrm{Na}^{+}$counterions are adsorbed at the exposed -OH groups and the carbonate ion is in contact with these two adsorbed ions, making two ionic bridges with the surface. In the case of the (100) plane, we observe a similar result. As shown by the density profile (Figure 5k), there is a depletion of carbonate near the surface. The configuration of maximum approach is also illustrated in the snapshot of Figure 5 $\mathrm{d}$. The carbonate is not in direct contact with the surface, but rather there is a $\mathrm{Na}^{+}$counterion making a bridge between 320 carbonate and the surface. This $\mathrm{Na}^{+}$counterions in contact with the surface, is in contact with one of the surface -OH groups (recall here that these -OH groups are not exposed to the interface in the crystal structure but modify their orientation in contact with water, see Figures $2 \mathrm{~d}$ and $3 \mathrm{~d}$ ).

In the case of $\mathrm{Ph}_{4} \mathrm{~B}^{-}$, we observe a weak attraction towards both surfaces, 325 as indicated by an increased density profile near the surface Figure 6. The configuration of maximum approach between the $\mathrm{Ph}_{4} \mathrm{~B}^{-}$ion and the surfaces is illustrated in the snapshots shown in Figure 6. In the case of the (010) surface, the orientation of the ion is influenced by the rugosity of the surface. This surface has "bumps" and also "holes" in which cellulose rings are exposed to 330 water. The $\mathrm{Ph}_{4} \mathrm{~B}^{-}$ion at the (010) surface is oriented in a way that corresponds to one of the phenyl rings inserted into one of these "holes" defined at the surface (see Figure 6p). In the case of the (100) surface, the configuration of maximum approach between the ion and the surface corresponds to one of the rings in contact with the surface (Figure 6 $\mathrm{d}$ ).

335 Therefore, the simultaneous hydrophilic and lipophilic behavior of the cellulose surface (with a smaller contact angle for the organic solvent), translates into a weak repulsion of hydrophilic ions and a weak attraction of hydrophobic ions.

Now, we consider the interaction of both cellulose surfaces with a protein 340 (simulations S9a, S9b, S10a and S10b in Table 2). We considered the case of the human Epidermal Growth Factor protein (hEGF), which could be of interest in potential uses of cellulose for tissue repair. The protein structure employed in the simulations was obtained from solution NMR at neutral $\mathrm{pH}$, deposited in the Protein Data Bank under PDB code 2KV4 [54] (see Appendix

345 for further details). At neutral $\mathrm{pH}$, the protein has a charge of $-5 \mathrm{e}$, according to the protonation states obtained using PropKa [55. In the simulations, we 

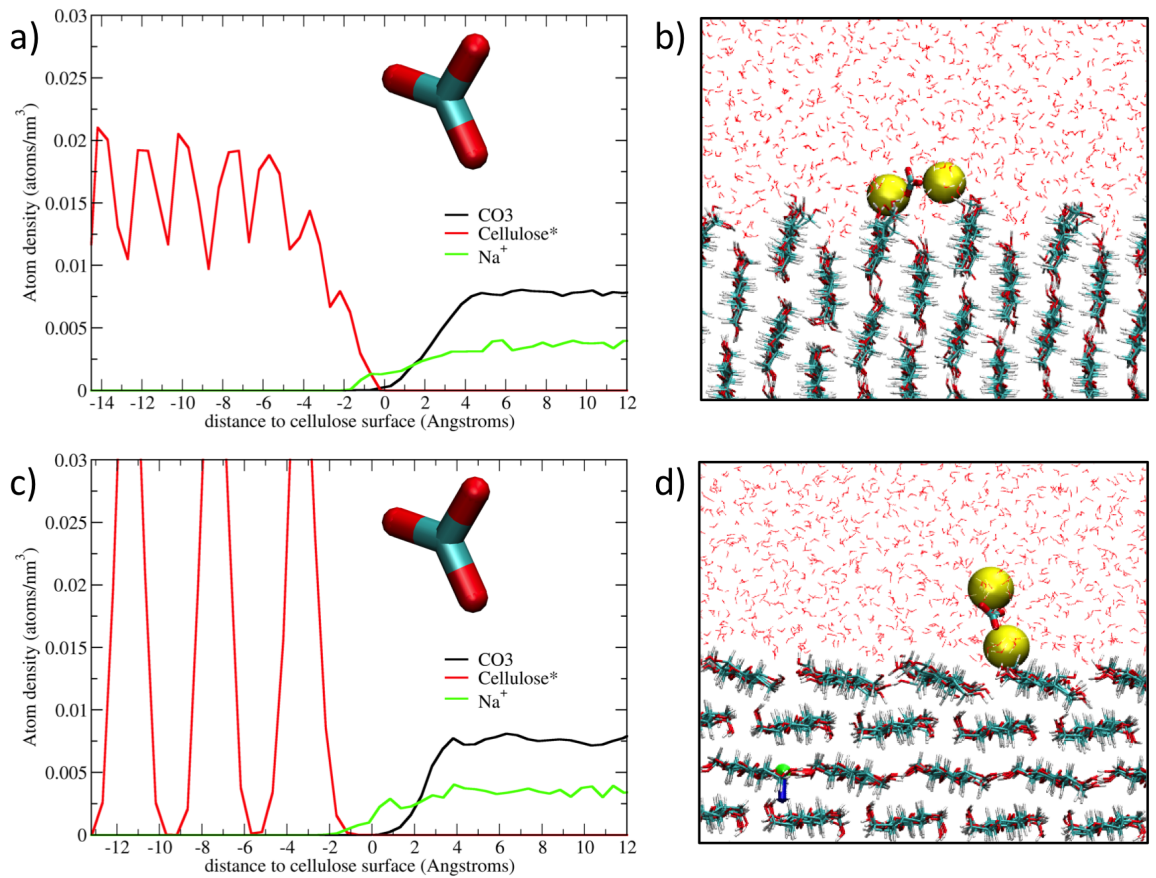

Figure 5: Cellulose interaction with carbonate ion. a) Atomic number density profile of carbonate (including all atoms) and $\mathrm{Na}^{+}$counter ions as a function of the distance to surface of cellulose plane (010). b) Snapshot of carbonate interacting with plane (010). $\mathrm{Na}^{+}$counter ions are shown in vdW representation.c) Atomic number density profile of carbonate (including all atoms) and $\mathrm{Na}^{+}$counter ions as a function of the distance to surface of cellulose plane (100). d) Snapshot of carbonate interacting with plane (100). $\mathrm{Na}^{+}$counter ions are shown in vdW representation. All density profiles for carbonate ion are calculated including all the atoms of the molecule. We include the atom density for cellulose to appreciate the proximity with the surface. We only included $\mathrm{O}$ and $\mathrm{C}$ atoms in this density calculation for cellulose and cellulose density was divided by a factor of 400 in order to appear in a similar scale.

consider $5 \mathrm{Na}^{+}$counterions to neutralize the system.

The results (Figure 7) show that in both planes (010) and (100) the protein a some point in the simulation prefers to be at the cellulose surface rather than to be in solution. In our simulations, hEGF protein was placed at $0.7 \mathrm{~nm}$ of distance of both cellulose planes as starting configuration. We considered two different, opposite orientations $a$ and $b$, indicated as a suffix in simulation names of Table 2. In orientation $a$ the protein is oriented with its long axis parallel to the surface and with the residues ASN1 and LEU52 placed in front of the surface. In orientation $b$ the protein is rotated $180^{\circ}$ on the longitudinal direction such as ASN1 and LEU52 are opposite to the surface. During a short equilibration run of a few ns, the protein moved to contact with the surface in all simulations (S9a, S9b, S10a and S10b), exploring the surface and changing its orientation depending on the exposed crystal plane. Protein adsorption shows substantial 

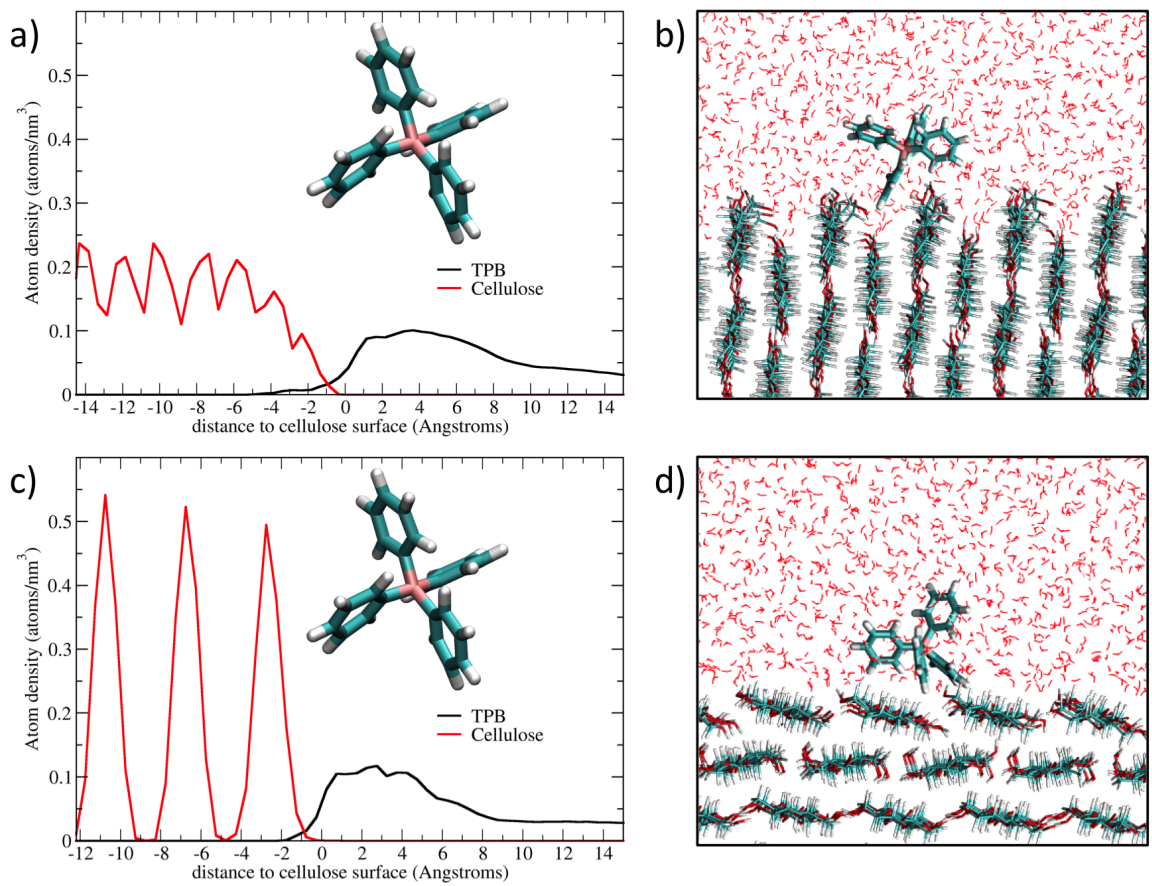

Figure 6: Cellulose interaction with tetraphenyl borate ion $\left(\mathrm{Ph}_{4} \mathrm{~B}^{-}\right)$. a) Atom density profile of $\mathrm{Ph}_{4} \mathrm{~B}^{-}$as function to the distance to surface of cellulose plane (010). b) Snapshot of $\mathrm{Ph}_{4} \mathrm{~B}^{-}$ interacting with plane (010).c) Atom density profile of $\mathrm{Ph}_{4} \mathrm{~B}^{-}$as function to the distance to surface of cellulose plane (100). d) Snapshot of $\mathrm{Ph}_{4} \mathrm{~B}^{-}$interacting with plane (100). All density profiles for $\mathrm{Ph}_{4} \mathrm{~B}^{-}$ion are calculated including all the atoms of the molecule. We include the atom density for cellulose to appreciate the proximity with the surface. We only included $\mathrm{O}$ and $\mathrm{C}$ atoms in cellulose density calculation and cellulose density was divided by a factor of 40 in order to appear in a similar scale.

differences between cellulose surface (010) and (100), as we will discuss next. At this point, we note that in ref [29] the authors identify an extended hydration layer that can preclude the approach of proteins (enzymes) to cellulose but we do not observe any evidence for this effect in our simulations.

Let us first discuss the results for simulation S9a and S9b (Figure 7a) corresponding to adsorption at the cellulose cellulose surface (010). In simulation S9a, hEGF protein binds at the very begging of simulation (during the equilibration run) and remains adsorbed during all the production run, as seen in Figure 7 a (black line). In simulation S9b (which considers an initial configuration of the protein rotated $180^{\circ}$ with respect to S9a) the hEGF protein explores the 370 surface before adsorbing, changing its relative orientation towards the surface. Finally, it adsorbs at the cellulose surface with the same orientation as obtained in simulation S9a and it remains adsorbed during 40 ns. The protein adsorption to cellulose plane (010) in both simulations S9a and S9b takes place through the same regions of the hEGF protein, which are two coils determined by the 


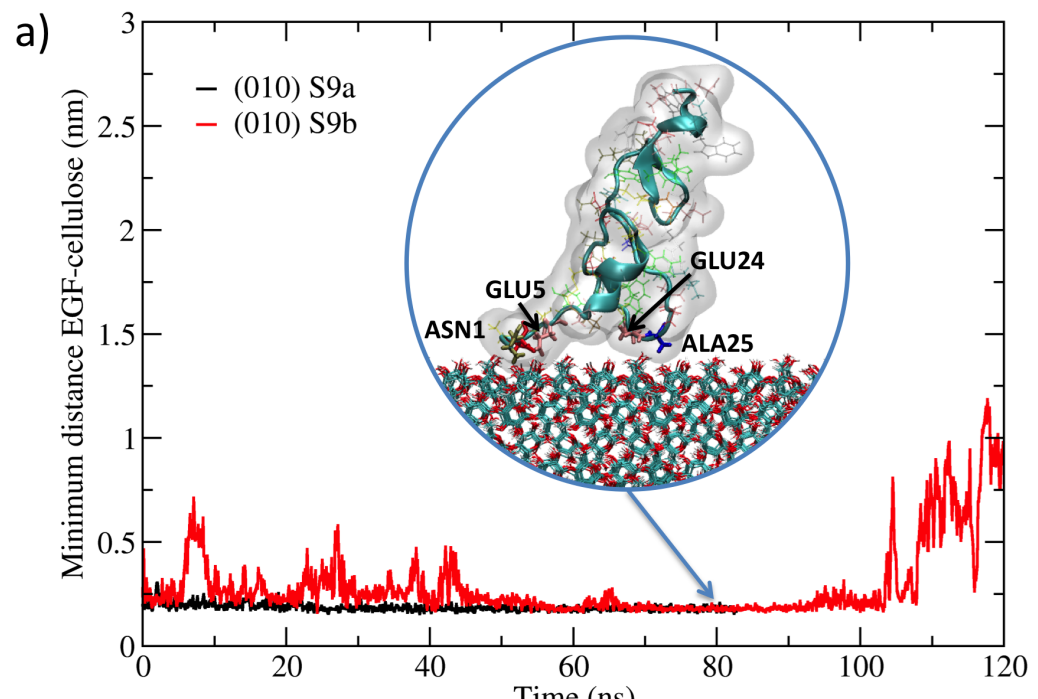

b)

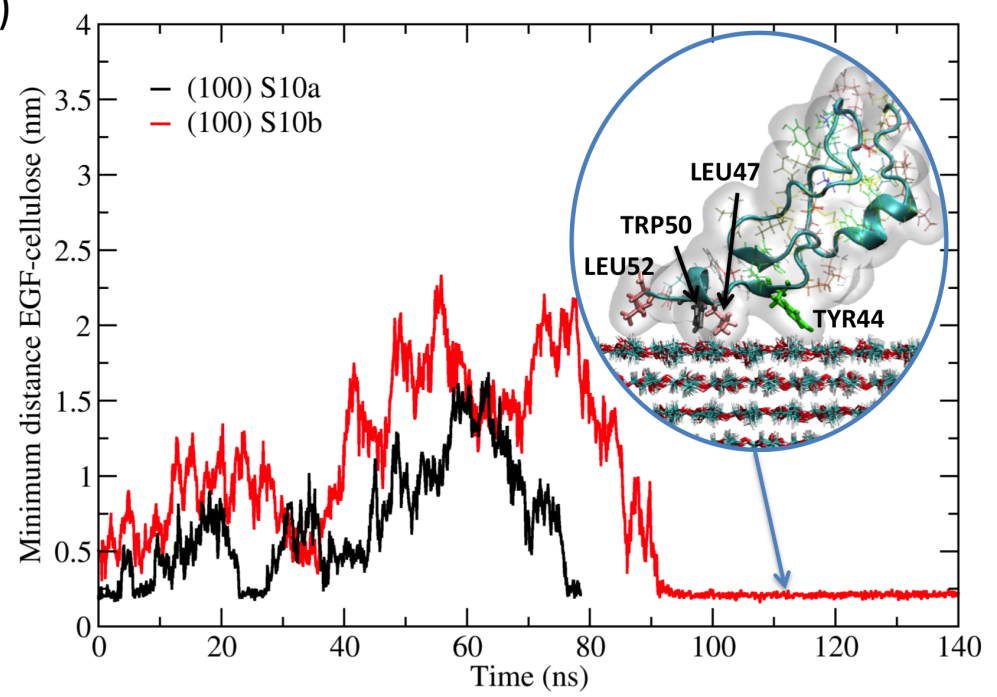

Figure 7: a) Plot of minimum distance between hEGF protein and cellulose plane (010) vs simulation time for simulations S9a and S9b (see Table 2). Inset: Snapshot of simulation S9b with hEGF protein adsorbed at cellulose surface (010) at simulation time of $80 \mathrm{~ns}$. The residues within $3.7 \AA$ of the surface are indicated in the inset. b) Plot of minimum distance between hEGF protein and cellulose plane (100) vs simulation time for simulations S10a and S10b (see Table 2). Inset: Snapshot of simulation S10b with hEGF protein adsorbed at cellulose surface (100) at simulation time of $113 \mathrm{~ns}$. The residues within $3.7 \AA$ of the surface are indicated in the inset. The structure of the hEGF protein appears in cartoon representation and the protein surface was also indicated as obtained from the surface algorithm implemented in VMD [16. 
375 aminoacids ASN1, SER2, ASP3, SER4, GLU5 and ILE23, GLU24, ALA25 and LEU26. At a given instant of time, we typically observe four of these aminoacids in contact with the cellulose surface, as shown in the inset of Figure $7 \mathrm{a}$ ) for a particular time. We recall here that this list of aminoacids involved in protein adsorption at the cellulose surface include both polar aminoacids (that interact

380 by hydrogen bonding) and nonpolar aminoacids (that interact by hydrophobic contacts). This is consistent with the results of the previous section that show a simultaneous hydrophilic and hydrophobic behaviour of this cellulose surface. The binding free energy associated with the interaction of these regions was estimated by Adaptive Bias Force method (ABF), as described in the Appendix.

${ }_{385}$ We obtain a value of $30 \mathrm{Kcal} / \mathrm{mol}$ per each of the two above mentioned coil regions, which corresponds to an average of $15 \mathrm{Kcal} / \mathrm{mol}$ per adsorbed residue.

In the case of adsorption onto the cellulose surface (100) (simulation S10a and S10b in Table 2), the protein remains at the cellulose surface or close to the cellulose surface without binding to the cellulose surface during a substantial simulation time, as seen in Figure 7b. After exploring the surface, in simulation S10b we observe the adsorption of the protein onto the surface and the protein remains adsorbed until the end of the simulation. The contact of the protein with the cellulose plane is mostly due to the apolar LEU52, TRP50, LEU47 and TYR44 residues, as shown in $7 \mathrm{~b}$ ). We also observe some configurations in

395 which in addition to these residues, the protein also has one or two residues of the ASN1, SER2, ASP3, SER4, GLU5 tail adsorbed onto the celulose surface. Our results show that the interaction of the hEGF protein with the (100) cellulose surface is mainly hydrophobic. These results are also consistent with previous results [30] which considered an extensive analysis of the adsorption of different aminoacids, dipeptides and tripeptides on plane (100) I $\beta$. In Ref 30 it was found that aminoacids with aromatic side chains are more prone to interact with plane (100) that have most of the hydrogen bond donors buried. We have estimated the free energy of adsorption of the hEGF protein to plane (100) (see Appendix for details), obtaining $\approx 150 \mathrm{Kcal} / \mathrm{mol}$. This large value of free energy

405 for interaction with plane (100) includes not only the interaction with LEU52, TRP50, LEU47 and TYR44 apolar residues but also the interaction of this cellulose plane with the tail formed by the residues ASN1, SER2, ASP3, SER4 and GLU5 which tries to approach to the surface as we detach the hydrophobic residues during the $\mathrm{ABF}$ biased simulation.

Therefore, the particular surface of cellulose involved in the interaction plays an important role in protein adsorption, with the hEGF protein being able to bind to the cellulose surface by different regions depending on the exposed crystallographic plane.

\section{Conclusion and Discussion}

415 In this study we have analyzed interactions of different molecules with cellulose crystal planes (100) and (010) of cellulose I $\beta$. These cellulose plane have very different chemical structure since plane (100) have most of the hydrogen bond donor groups buried in the surface, while plane (010) have most of this 
groups exposed. Other crystallographic planes will have an intermediate character between (100) and (010) so they will show an intermediate character in their interactions with solvents and molecules 24. The conclusions obtained from our MD simulations of $\mathrm{I} \beta$ cellulose nanocrystals can be summarized as follows:

- The obtained contact angles for water and tetradecane onto (010) and (100) surfaces of I $\beta$ cellulose indicate that both surfaces are simultaneously hydrophilic and lipophilic. This result is remarkable considering the very different hydrogen bonding capabilities of the two surfaces and their different exposure of hydrophobic groups at the surface.

- Wetting of the cellulose surface is strongly heterogeneous at the molecular scale in the case of both water and organic solvents. This complex surface behavior suggests the possibility of new and interesting applications for the use of nanocellulose materials which will deserve further modeling studies.

- Both hydrophilic and hydrophobic ions have a weak interaction with nanocellulose surfaces (although hydrophobic ions seem to have a somewhat stronger interaction with cellulose than hydrophilic ones), a result which can be attributed to the simultaneous hydrophilic and lipophilic behavior of cellulose surfaces.

The soluble hEGF protein binds to both the (010) and (100) cellulose surface through different regions of the protein which contain both polar and apolar residues. In the case of the (010) surface, the aminoacids involved in adsorption are mostly polar aminoacids whereas in the case of adsorption at the (100) surface most of the involved aminoacids are apolar. This result suggests that the simultaneous hydrophilic and hydrophobic character of cellulose induces a substantial interaction of cellulose with proteins (which contain both polar and apolar aminoacids available for interaction with substrates).

Finally, it is important to note that our conclusions are based on the study of a untreated crystal cellulose surface based on crystallographic data. But real nanocellulose based materials are expected to be even more complex. For example, measurements by different groups systematically indicate that cellulose is negatively charged under neutral $\mathrm{pH}$, with an iso-electric point of 3.7 [43], a result completely unexpected from the chemical structure of an unmodified cellulose crystal. This is a clear indication of the presence of acid groups at the surface of cellulose. Measurements on pure cellobiose show that the molecule ${ }_{455}$ is neutral below a $\mathrm{pH}$ of about 10.5, in agreement with theoretical calculations from molecular structure [28. It seems plausible that these acid groups present at cellulose surface are due to surface modifications on the cellulose nano-fibrils produced during its bio-synthesis. Some of the most common synthesis methods of cellulose nanoparticles are believed to induce the presence of surface groups such as sulfate ester groups (sulfuric acid hydrolysis), hydroxyl 
groups (hydrochloric acid hydrolysis), acetyl groups (acetic acid hydrolysis), carboxylic acid groups (carboxymethylation route) and others [56]. Thus, modeling and simulation of the interactions of real cellulose materials with solvents and molecules requires to include these chemical surface groups in the model, which means that models should take into account the synthesis route of the cellulose. The appropriate way to modify present models (based on crystal structure) to include these surface chemical groups and its dependence on the synthesis route in the models is far from obvious, but it is clear that this should be the next step in theoretical studies involving the modeling of cellulose.

\section{Acknowledgments}

We acknowledge financial support from the Spanish Government through the MAT2015-64442-R grant and the Severo Ochoa Grant SEV-2015-0496 for Research Centres of Excellence awarded to ICMAB. D.C. Malaspina is supported by the European Union's Horizon 2020 research and innovation programme 475 under Marie Sklodowska-Curie grant agreement No 6655919. The simulations reported here were performed using the Edison Supercomputing facility of the National Energy Research Scientific Computing Center (NERSC), a U.S. Department of Energy Office of Science User Facility operated under Contract No. DE-AC02-05CH11231. Useful discussions with Prof. A. Roig and Dr A. Laromaine from the Nanoparticles and Nanocomposites Group at ICMAB are acknowledged.

\section{Appendix: Simulation Methods}

Molecular dynamics (MD) simulations were performed using NAMD 2.12 software [57. All simulation snapshots were made using Visual Molecular Dy485 namics (VMD) software [16]. Cellulose crystal planes were build using the Cellulose builder toolkit [15] and described using the carbohydrate section of the CHARMM36 force field [37. In all simulations, water was described employing the TIP4P/2005 model [40, 42] which best capture hydrogen-bonding features of liquid water at all pressures [41. Tetraphenil borate, tetradecane, carbonate and methane were described by CHARMM36 general force field (CGenFF) 38. Human epidermal growth factor protein (hEGF) structure was obtained from the Protein Data Bank (PDB code:2KV4) [54]. The force field employed for the protein was the CHARMM36 force field protein section [58. The composition, simulation box size, simulation time and other details for all simulated systems are described in Tables 1 and 2 . In all simulations we employed periodic boundary conditions in all directions. The protocol followed in all simulations includes an initial minimization, equilibration and production runs. In all simulations we control temperature with a Langevin thermostat at $298 \mathrm{~K}$ using a damping coefficient of $1 \mathrm{ps}^{-1}$. Simulations of wetting by a solvent droplet (S1-S4 in Table $\left.{ }_{500} 11\right)$ were made in the NVT ensemble. Simulations of interaction of cellulose with molecules (s5-S10 in Table 22) were made in the NPT ensemble. The barostat 
employed in NPT simulations was a Langevin piston kept at 1 atm with a piston period of $100 \mathrm{fs}$ and piston decay of $50 \mathrm{fs}$. The pressure was applied in the direction of the water slab ( $z$ direction) using the anisotropic barostat option of NAMD. In these simulations, the dimensions of the simulation box in $x$ and $y$ directions was maintained constant. In all simulations Newton's equation of motion were integrated every $2 \mathrm{fs}$ and electrostatic interactions updated every 4 fs. All bonds between heavy atoms and hydrogen atoms were maintained rigid using rigid bonds. Short range electrostatic and Lennard-Jones potential 510 was computed with a cutoff of $1.2 \mathrm{~nm}$ and a switching distance of $1.0 \mathrm{~nm}$. For long range electrostatic we used Particle Mesh Ewald (PME) algorithm using a grid spacing of $1.0 \mathrm{~nm}$. In all simulations we added $\mathrm{Na}^{+}$ions to obtain electroneutrality of the system. For systems S9 and S10 of Table2, hEGF protein was placed initially at $0.7 \mathrm{~nm}$ from the cellulose surface during the equilibration run, 515 but during the production run the protein is initially near the surface. For the calculation of density profiles in Figure 5 and in Figure 5 we implement our own Fortran code and for the 2-D water density of Figure 3 we implemented our own code in Python. The value of contact angle was approximated by superposition of different trial angles over the density profile of Figure 3. The minimum dis${ }_{520}$ tance in Figure 7 was calculated using gmx mindist tool from GROMACS [59]. For simulations S9b and S10b we have performed free-energy calculations using the Adaptive Biasing Force (ABF) technique as implemented in NAMD 60. In these biased MD simulations we obtain the potential of mean force (PMF) for the interaction of hEGF from cellulose. We selected as a reaction coordinate ${ }_{525}$ the z-axis projection of the distance between the center of mass of the cellulose surface and the center of mass of a group of protein residues involved in the protein adsorption. The force constant employed in the ABF calculation was 10 kcal mol1 2 and the PMF was obtained with 0.1 resolution. In the case of the PMF calculation for the S9B simulation, we start from a configuration with an 530 initially adsorbed protein over the cellulose surface (as obtained from simulation $\mathrm{S9B})$. We performed the ABF simulation by considering as generalized coordinate the center of mass distance between cellulose and the aminoacids 23, 24, 25 and 26 of the protein aminoacid sequence, which correspond to one of the two coils of the protein initially adsorbed to cellulose. Once these aminoacids were 535 detached from the surface, we performed a second ABF simulation in which the generalized coordinate was the center of mass distance between cellulose and the aminoacids 1, 2, 3 and 4, which corresponds to the other coil of the protein that adsorbs over cellulose. In this way, the protein was fully detached from the surface. In the case of the PMF calculation for the S10B simulation, we considered as generalized coordinate the center of mass distance between cellulose and the aminoacids $43,44,47,50,52,15$ and 8 of the protein aminoacid sequence, which correspond to the region of the protein initially adsorbed to cellulose. In this case, we find that the convergence of the ABF simulations to obtain a well defined PMF were difficult, so we decided to perform two different, complemen-

545 tary ABF simulations. In the first one, we started from a configuration with an initially adsorbed protein over the cellulose surface, as obtained from simulation S10B. In the second ABF simulation, we reversed the previous ABF simulation, 
starting from an initially desorbed protein and ending with the protein adsorbed onto cellulose. The two simulations produced almost identical PMF values.

6 ] R. J. Moon, A. Martini, J. Nairn, J. Simonsen, J. Youngblood, Cellulose nanomaterials review: structure, properties and nanocomposites, Chemical Society Reviews 40 (7) (2011) 3941-3994. doi:10.1039/c0cs00108b URL http://dx.doi.org/10.1039/c0cs00108b

7] B. Lindman, From surfactant to cellulose and DNA self-assembly. A 50year journey, Colloid and Polymer Science 294 (11) (2016) 1687-1703. doi : 10.1007/s00396-016-3927-2.

URL http://dx.doi.org/10.1007/s00396-016-3927-2

[8] M. Mart'ınez-Sanz, M. J. Gidley, E. P. Gilbert, Hierarchical architecture of bacterial cellulose and composite plant cell wall polysaccharide hydrogels using small angle neutron scattering, Soft Matter 12 (5) (2016) 1534-1549. doi:10.1039/c5sm02085a. URL http://dx.doi.org/10.1039/c5sm02085a 
[9] T. Heinze, O. A. El Seoud, A. Koschella, Cellulose derivatives : synthesis, structure, and properties, Springer Series on Polymer and Composite Materials, 2018. URL http://www .worldcat.org/isbn/9783319731681

[10] B. Medronho, B. Lindman, Brief overview on cellulose dissolution/regeneration interactions and mechanisms, Advances in Colloid and Interface Science 222 (2015) 502-508. doi:10.1016/j . cis.2014.05.004. URL http://dx.doi.org/10.1016/j.cis.2014.05.004

[11] L. Alves, B. F. Medronho, F. E. Antunes, A. Romano, M. G. Miguel, n B. Lindman, On the role of hydrophobic interactions in cellulose dissolution and regeneration: Colloidal aggregates and molecular solutions, Colloids and Surfaces A: Physicochemical and Engineering Aspects 483 (2015) 257263. doi:10.1016/j.colsurfa.2015.03.011. URL http://dx.doi.org/10.1016/j.colsurfa.2015.03.011

[12] L. Alves, B. Medronho, F. Antunes, D. Topgaard, B. Lindman, Dissolution state of cellulose in aqueous systems. 1. Alkaline solvents, Cellulose 23 (1) (2016) 247-258. doi:10.1007/s10570-015-0809-6. URL http://dx.doi.org/10.1007/s10570-015-0809-6

[13] W. Glasser, R. Atalla, J. Blackwell, R. Malcolm Brown, W. Burchard, A. French, D. Klemm, Y. Nishiyama, About the structure of cellulose: debating the Lindman hypothesis, Cellulose 19 (3) (2012) 589-598. doi: 10.1007/s10570-012-9691-7. URL http://dx.doi.org/10.1007/s10570-012-9691-7

610 [14] S.-Y. Ding, M. E. Himmel, The Maize Primary Cell Wall Microfibril: A New Model Derived from Direct Visualization, Journal of Agricultural and Food Chemistry 54 (3) (2006) 597-606. doi:10.1021/jf051851z. URL http://dx.doi.org/10.1021/jf051851z

[15] T. C. F. Gomes, M. S. Skaf, Cellulose-Builder: A toolkit for building crystalline structures of cellulose, Journal of Computational Chemistry 33 (14) (2012) 1338-1346. doi:10.1002/jcc.22959.

URL http://dx.doi.org/10.1002/jcc.22959

[16] W. Humphrey, A. Dalke, K. Schulten, VMD: Visual molecular dynamics, Journal of Molecular Graphics 14 (1) (1996) 33-38. doi:10.1016/ 0263-7855(96) 00018-5. URL http://dx.doi.org/10.1016/0263-7855(96)00018-5

[17] D. Frenkel, B. Smit, Understanding Molecular Simulation, 2nd Edition, Academic Press, Inc., Orlando, FL, USA, 2001.

[18] J. H. Jensen, Molecular modeling basics, CRC Press, 2010. URL http://www . worldcat . org/isbn/9781420075267 
[19] H. Miyamoto, U. Schnupf, M. F. Crowley, J. W. Brady, Comparison of the simulations of cellulosic crystals with three carbohydrate force fields,

Carbohydrate Research 422 (2016) 17-23. doi:10.1016/j.carres.2016. 01.001

URL http://dx.doi.org/10.1016/j.carres.2016.01.001

[20] S. Roig-Sanchez, E. Jungstedt, I. Anton-Sales, D. C. Malaspina, J. Faraudo, L. A. Berglund, A. Laromaine, A. Roig, Nanocellulose films with multiple functional nanoparticles in confined spatial distribution, Nanoscale Horizonsdoi:10.1039/c8nh00310f URL http://dx.doi.org/10.1039/c8nh00310f

[21] C. A. López, G. Bellesia, A. Redondo, P. Langan, S. P. S. Chundawat, B. E. Dale, S. J. Marrink, S. Gnanakaran, MARTINI Coarse-Grained Model for Crystalline Cellulose Microfibers, J. Phys. Chem. B 119 (2) (2015) 465-473. doi:10.1021/jp5105938.

640 URL http://dx.doi.org/10.1021/jp5105938

[22] A. Poma, M. Chwastyk, M. Cieplak, Coarse-grained model of the native cellulose Ialpha and the transformation pathways to the Ibeta allomorph, Cellulose 23 (3) (2016) 1573-1591. doi:10.1007/s10570-016-0903-4 URL http://dx.doi.org/10.1007/s10570-016-0903-4

[23] Y. Nishiyama, G. P. Johnson, A. D. French, V. T. Forsyth, P. Langan, Neutron Crystallography, Molecular Dynamics, and Quantum Mechanics Studies of the Nature of Hydrogen Bonding in Cellulose I , Biomacromolecules 9 (11) (2008) 3133-3140. doi:10.1021/bm800726v. URL http://dx.doi.org/10.1021/bm800726v

[24] K. Mazeau, On the external morphology of native cellulose microfibrils, 1] Carbohydrate Polymers 84 (1) (2011) 524-532. doi:10.1016/j.carbpol. 2010.12.016 URL http://dx.doi.org/10.1016/j.carbpol.2010.12.016

[25] M. Bergenstråhle, L. A. Berglund, K. Mazeau, Thermal Response in Crystalline I Cellulose: A Molecular Dynamics Study, J. Phys. Chem. B 111 (30) (2007) 9138-9145. doi:10.1021/jp072258i. URL http://dx.doi.org/10.1021/jp072258i

[26] J. F. Matthews, G. T. Beckham, M. Bergenstråhle-Wohlert, J. W. Brady, M. E. Himmel, M. F. Crowley, Comparison of Cellulose I Simulations with Three Carbohydrate Force Fields, Journal of Chemical Theory and Computation 8 (2) (2012) 735-748. doi:10.1021/ct2007692. URL http://dx.doi.org/10.1021/ct2007692

[27] M. Bergenstråhle, J. Wohlert, M. E. Himmel, J. W. Brady, Simulation studies of the insolubility of cellulose, Carbohydrate Research 345 (14)

(2010) 2060-2066. doi:10.1016/j.carres.2010.06.017. URL http://dx.doi.org/10.1016/j.carres.2010.06.017 
[28] E. Bialik, B. Stenqvist, Y. Fang, A. Östlund, I. Furó, B. Lindman, M. Lund, D. Bernin, Ionization of Cellobiose in Aqueous Alkali and the Mechanism of Cellulose Dissolution, J. Phys. Chem. Lett. 7 (24) (2016) 5044-5048. doi:10.1021/acs.jpclett.6b02346 URL http://dx.doi .org/10.1021/acs .jpclett.6b02346

[29] J. F. Matthews, C. E. Skopec, P. E. Mason, P. Zuccato, R. W. Torget,

n J. Sugiyama, M. E. Himmel, J. W. Brady, Computer simulation studies of microcrystalline cellulose I, Carbohydrate Research 341 (1) (2006) 138152. doi:10.1016/j.carres.2005.09.028.

URL http://dx.doi.org/10.1016/j.carres.2005.09.028

[30] G. Nawrocki, P.-A. Cazade, D. Thompson, M. Cieplak, Peptide Recognition Capabilities of Cellulose in Molecular Dynamics Simulations, The Journal

1. of Physical Chemistry C 119 (43) (2015) 24404-24416. doi:10.1021/acs. jpcc.5b07118.

URL http://dx.doi.org/10.1021/acs.jpcc.5b07118

[31] K. Mazeau, A. Rivet, Wetting the (110) and (100) Surfaces of I Cellulose Studied by Molecular Dynamics, Biomacromolecules 9 (4) (2008) 13521354. doi:10.1021/bm7013872. URL http://dx.doi.org/10.1021/bm7013872

[32] R. J. Maurer, A. F. Sax, V. Ribitsch, Molecular simulation of surface reorganization and wetting in crystalline cellulose I and II, Cellulose 20 (1) (2013) 25-42. doi:10.1007/s10570-012-9835-9. URL http://dx.doi.org/10.1007/s10570-012-9835-9

690 [33] H. Miyamoto, D. M. Rein, K. Ueda, C. Yamane, Y. Cohen, Molecular dynamics simulation of cellulose-coated oil-in-water emulsions, Cellulose 24 (7) (2017) 2699-2711. doi:10.1007/s10570-017-1290-1. URL http://dx.doi.org/10.1007/s10570-017-1290-1

[34] M. Bergenstråhle, E. Thormann, N. Nordgren, L. A. Berglund, Force 695 Pulling of Single Cellulose Chains at the Crystalline CelluloseLiquid Interface: A Molecular Dynamics Study, Langmuir 25 (8) (2009) 4635-4642. doi:10.1021/la803915c.

URL http://dx.doi.org/10.1021/la803915c

[35] P. Chen, Y. Nishiyama, J. Wohlert, A. Lu, K. Mazeau, A. E. Ismail, Trans$700 \quad$ lational Entropy and Dispersion Energy Jointly Drive the Adsorption of Urea to Cellulose, The Journal of Physical Chemistry B 121 (10) (2017) 2244-2251. doi:10.1021/acs.jpcb.6b11914. URL http://dx.doi.org/10.1021/acs .jpcb.6b11914

[36] R. Alqus, S. J. Eichhorn, R. A. Bryce, Molecular Dynamics of Cellulose 705 Amphiphilicity at the GrapheneWater Interface, Biomacromolecules 16 (6) (2015) 1771-1783. doi:10.1021/acs.biomac.5b00307. URL http://dx.doi .org/10.1021/acs.biomac.5b00307 
[37] O. Guvench, S. S. Mallajosyula, E. P. Raman, E. Hatcher, K. Vanommeslaeghe, T. J. Foster, F. W. Jamison, A. D. MacKerell, CHARMM Ad-

10 ditive All-Atom Force Field for Carbohydrate Derivatives and Its Utility in Polysaccharide and CarbohydrateProtein Modeling, Journal of Chemical Theory and Computation 7 (10) (2011) 3162-3180. doi:10.1021/ ct200328p.

URL http://dx.doi.org/10.1021/ct200328p

[38] K. Vanommeslaeghe, E. Hatcher, C. Acharya, S. Kundu, S. Zhong, J. Shim, E. Darian, O. Guvench, P. Lopes, I. Vorobyov, A. D. Mackerell, CHARMM general force field: A force field for drug-like molecules compatible with the CHARMM all-atom additive biological force fields, Journal of Computational Chemistry 31 (4) (2009) NA. doi:10.1002/jcc.21367. URL http://dx.doi.org/10.1002/jcc.21367

[39] A. D. MacKerell, D. Bashford, M. Bellott, R. L. Dunbrack, J. D. Evanseck, M. J. Field, S. Fischer, J. Gao, H. Guo, S. Ha, D. Joseph-McCarthy, L. Kuchnir, K. Kuczera, F. T. K. Lau, C. Mattos, S. Michnick, T. Ngo, D. T. Nguyen, B. Prodhom, W. E. Reiher, B. Roux, M. Schlenkrich, J. C.

${ }_{725}$ Smith, R. Stote, J. Straub, M. Watanabe, J. Wiórkiewicz-Kuczera, D. Yin,

n. Karplus, All-Atom Empirical Potential for Molecular Modeling and Dynamics Studies of Proteins †, The Journal of Physical Chemistry B 102 (18) (1998) 3586-3616. doi:10.1021/jp973084f. URL http://dx.doi.org/10.1021/jp973084f

730 [40] J. L. F. Abascal, C. Vega, A general purpose model for the condensed phases of water: TIP4P/2005, The Journal of Chemical Physics 123 (23) (2005) $234505+$. doi:10.1063/1.2121687. URL http://dx.doi.org/10.1063/1.2121687

[41] C. Calero, J. Martí, E. Guàrdia, 1H Nuclear Spin Relaxation of Liquid Water from Molecular Dynamics Simulations, J. Phys. Chem. B 119 (5) (2015) 1966-1973. doi:10.1021/jp510013q. URL http://dx.doi.org/10.1021/jp510013q

[42] C. Vega, J. L. F. Abascal, Simulating water with rigid non-polarizable models: a general perspective, Physical Chemistry Chemical Physics 13 (44) (2011) 19663-19688. doi:10.1039/c1cp22168j. URL http://dx.doi.org/10.1039/c1cp22168j

[43] K.-Y. Lee, F. Quero, J. Blaker, C. Hill, S. Eichhorn, A. Bismarck, Surface only modification of bacterial cellulose nanofibres with organic acids, Cellulose 18 (3) (2011) 595-605. doi:10.1007/s10570-011-9525-z.

745 URL http://dx.doi.org/10.1007/s10570-011-9525-z

[44] G. Morandi, L. Heath, W. Thielemans, Cellulose Nanocrystals Grafted with Polystyrene Chains through Surface-Initiated Atom Transfer Radical Polymerization (SI-ATRP), Langmuir 25 (14) (2009) 8280-8286. doi:10. 
1021/la900452a.

[45] M. Abdelmouleh, S. Boufi, M. N. Belgacem, A. P. Duarte, A. Ben Salah, A. Gandini, Modification of cellulosic fibres with functionalised silanes: development of surface properties, International Journal of Adhesion and Adhesives 24 (1) (2004) 43-54. doi:10.1016/s0143-7496(03) 00099-x. URL http://dx.doi.org/10.1016/s0143-7496(03) 00099-x

[46] G. I. Mantanis, R. A. Young, Wetting of wood, Wood Science and Technology 31 (5) (1997) 339-353. doi:10.1007/bf01159153. URL http://dx.doi.org/10.1007/bf01159153

[47] I. Kalashnikova, H. Bizot, B. Cathala, I. Capron, Modulation of Cellulose Nanocrystals Amphiphilic Properties to Stabilize Oil/Water Interface, Biomacromolecules 13 (1) (2012) 267-275. doi:10.1021/bm201599j. URL http://dx.doi.org/10.1021/bm201599j

[48] A. P. dos Santos, A. Diehl, Y. Levin, Surface Tensions, Surface Potentials, and the Hofmeister Series of Electrolyte Solutions, Langmuir 26 (13) (2010) 10778-10783. doi:10.1021/la100604k. URL http://dx.doi.org/10.1021/la100604k

[49] C. Calero, J. Faraudo, D. Bastos-González, Interaction of monovalent ions with hydrophobic and hydrophilic colloids: Charge inversion and ionic specificity, Journal of the American Chemical Society 133 (38) (2011) $15025-15035$.

[50] D. Bastos-González, L. Pérez-Fuentes, C. Drummond, J. Faraudo, Ions at interfaces: the central role of hydration and hydrophobicity, Current Opinion in Colloid \& Interface Science 23 (2016) 19-28.

[51] S. Yadav, A. Chandra, Structural and Dynamical Nature of Hydration 775 . Shells of the Carbonate Ion in Water: An Ab Initio Molecular Dynamics Study, The Journal of Physical Chemistry B 122 (4) (2018) 1495-1504. doi:10.1021/acs.jpcb.7b11636.

URL http://dx.doi.org/10.1021/acs.jpcb.7b11636

[52] L. Pérez-Fuentes, C. Drummond, J. Faraudo, D. Bastos-González, Anions make the difference: insights from the interaction of big cations and anions with poly (N-isopropylacrylamide) chains and microgels, Soft Matter 11 (25) (2015) 5077-5086.

[53] L. Pérez-Fuentes, C. Drummond, J. Faraudo, D. Bastos-González, Interaction of organic ions with proteins, Soft matter 13 (6) (2017) 1120-1131.

785 [54] H.-W. Huang, S. K. Mohan, C. Yu, The NMR solution structure of hua man epidermal growth factor (hEGF) at physiological $\mathrm{pH}$ and its interactions with suramin, Biochemical and Biophysical Research Communications 402 (4) (2010) 705-710. doi:10.1016/j.bbrc.2010.10.089. URL http://dx.doi.org/10.1016/j.bbrc.2010.10.089 
[55] H. Li, A. D. Robertson, J. H. Jensen, Very fast empirical prediction and rationalization of protein pKa values, Proteins: Structure, Function, and Bioinformatics 61 (4) (2005) 704-721. doi:10.1002/prot.20660. URL http://dx.doi.org/10.1002/prot.20660

[56] A. Dufresne, Nanocellulose: From Nature to High Performance Tailored Materials, De Gruyter textbook, De Gruyter, 2012. URL https://www.degruyter.com/viewbooktoc/product/129215

[57] J. C. Phillips, R. Braun, W. Wang, J. Gumbart, E. Tajkhorshid, E. Villa, C. Chipot, R. D. Skeel, L. Kalé, K. Schulten, Scalable molecular dynamics with NAMD, Journal of Computational Chemistry 26 (16) (2005) 17811802. doi:10.1002/jcc.20289. URL http://dx.doi.org/10.1002/jcc.20289

[58] R. B. Best, X. Zhu, J. Shim, P. E. M. Lopes, J. Mittal, M. Feig, A. D. MacKerell, Optimization of the Additive CHARMM All-Atom Protein Force Field Targeting Improved Sampling of the Backbone, and Side-Chain 1 and 2 Dihedral Angles, Journal of Chemical Theory and Computation 8 (9) (2012) 3257-3273. doi:10.1021/ct300400x. URL http://dx.doi.org/10.1021/ct300400x

[59] D. Van Der Spoel, E. Lindahl, B. Hess, G. Groenhof, A. E. Mark, H. J. C. Berendsen, GROMACS: Fast, flexible, and free, Journal of Computational Chemistry 26 (16) (2005) 1701-1718. doi:10.1002/jcc.20291 URL http://dx.doi.org/10.1002/jcc.20291

[60] J. Henin, G. Fiorin, C. Chipot, M. L. Klein, Exploring Multidimensional

․ Free Energy Landscapes Using Time-Dependent Biases on Collective Variables, Journal of Chemical Theory and Computation 6 (1) (2010) 35-47. ${ }_{815}$ doi:10.1021/ct9004432.

URL http://dx .doi .org/10.1021/ct9004432 